Engineering Physics and Mathematics Division

Mathematical Sciences Section

\title{
DOLIB: DISTRIBUTED OBJECT LIBRARY
}

\author{
E.F. D'Azevedo \\ C.H. Romine \\ Mathematical Sciences Section \\ Oak Ridge National Laboratory \\ P.O. Box 2008, Bldg. 6012 \\ Oak Ridge, TN 37831-6367
}

Date Published: October 1994

Research supported by the Applied Mathematical Sciences subprogram of the Office of Energy Research, U.S. Department of Energy

Prepared by the

Oak Ridge National Laboratory

Oak Ridge, Tennessee 37831

managed by

Martin Marietta Energy Systems, Inc.

for the

U.S. DEPARTMENT OF ENERGY

under Contract No. DE-AC05-84OR21400 


\section{Contents}

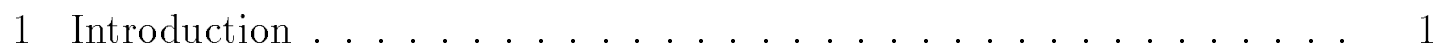







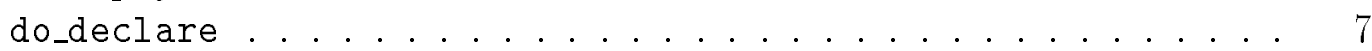

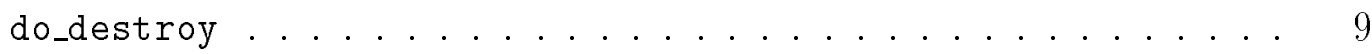



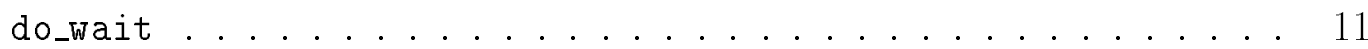

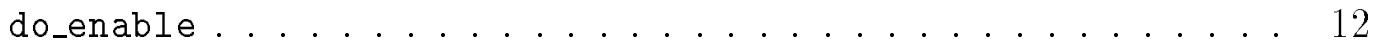

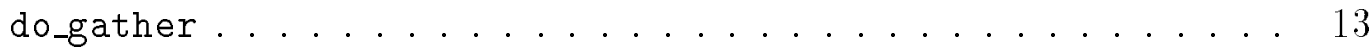



do_isavail ............................ 17







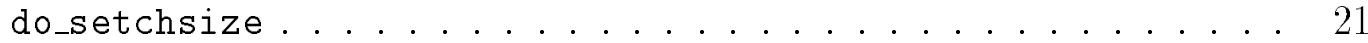

4 Implementation Details . . . . . . . . . . . . . 22

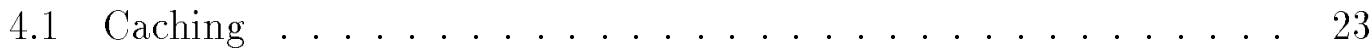

4.2 Accessing a Global Shared Array . . . . . . . . . . . 25

4.3 Reclaiming Memory . . . . . . . . . . . . . 26

5 Performance of DOLIB on Intel iPSC/860 . . . . . . . . . . . 26

6 Implementation of DOLIB using Polling . . . . . . . . . . 27

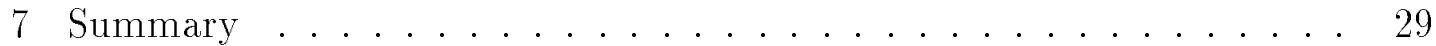

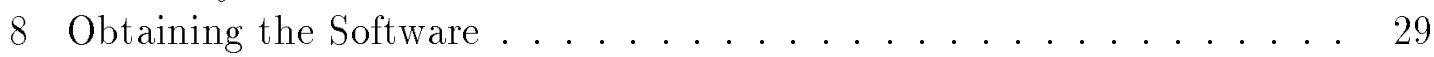



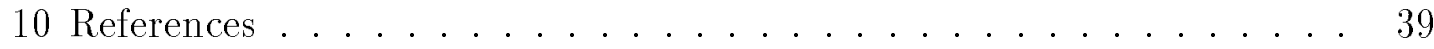




\title{
DOLIB: DISTRIBUTED OBJECT LIBRARY
}

\author{
E.F. D’Azevedo \\ C.H. Romine
}

\begin{abstract}
This report describes the use and implementation of DOLIB (Distributed Object Library), a library of routines that emulates global or virtual shared memory on Intel multiprocessor systems. Access to a distributed global array is through explicit calls to gather and scatter. Advantages of using DOLIB include: dynamic allocation and freeing of huge (gigabyte) distributed arrays, both $\mathrm{C}$ and FORTRAN callable interfaces, and the ability to mix shared-memory and message-passing programming models for ease of use and optimal performance. DOLIB is independent of language and compiler extensions and requires no special operating system support. DOLIB also supports automatic caching of read-only data for high performance. The virtual shared memory support provided in DOLIB is well suited for implementing Lagrangian particle tracking techniques. We have also used DOLIB to create DONIO (Distributed Object Network I/O Library), which obtains over a 10-fold improvement in disk I/O performance on the Intel Paragon.
\end{abstract}




\section{Introduction}

Distributed memory multiprocessors have proven to be scalable and offer high performance. However, the limited memory on each processor node generally requires programers to perform their own data decomposition, carefully moving needed data among nodes by explicit message passing. Writing parallel application code using message passing is both intricate and error prone. Virtual shared memory enables a programmer to make full use of the total aggregate (gigabyte) memory resources while avoiding the difficulties of message passing.

DOLIB (Distributed Object Library) supports virtual shared memory on the Intel Paragon and iPSC/860 distributed memory supercomputers. DOLIB enables all processors to operate directly on any part of a distributed global array through explicit calls to gather and scatter. Advantages of using DOLIB include: dynamic allocation and deallocation of huge (gigabyte) distributed arrays, both $\mathrm{C}$ and FORTRAN callable interfaces, and the ability to mix shared-memory and message-passing programming models for ease of use and optimal performance. DOLIB is independent of any language or compiler extensions, and requires no special operating system support. DOLIB supports automatic caching of read-only data for high performance.

Section 2 discusses previous work related to DOLIB. In section 3, we describe the user interface in the form of manual pages for the DOLIB procedures. In section 4, we provide implementation details of DOLIB, to aid the programmer in optimizing the use of DOLIB's global arrays. Section 5 illustrates the effectiveness and ease of use of DOLIB with a parallel matrix-matrix multiply. We have also used DOLIB to create DONIO, the Distributed Object Network I/O Library; details can be found in D'Azevedo and Romine [1]. DoNIo obtains more than a 10-fold improvement in performance of concurrent disk I/O on the Intel Paragon. Finally, section 7 gives a short summary and a preview of upcoming developments in future versions of DOLIB. 


\section{Previous Work}

The performance of virtual shared memory on a distributed memory system requires an effective caching strategy. Thus, much of the research into virtual shared memory, such as Li [2], Li and Hudak [3], and Stumm and Zhou [7], concerns intricate network protocols that maintain cache coherency in the presence of multiple concurrent updates.

Shiva [4] is a shared virtual memory system for the Intel iPSC/2 hypercube multiprocessor. Shiva uses the Memory Management Unit (MMU) page fault mechanism on each Intel i386 node to generate memory requests for remote pages. The implementation requires low level hardware and operating system support.

The CHAOS library [6] is an attempt to provide support for the parallel solution of irregular problems; that is, problems whose communication patterns are not easily predictable. CHAOS is a runtime library that can analyze the pattern of indirect addressing of arrays (such as $\mathrm{x}(\mathrm{ia}(\mathrm{i}))=\mathrm{x}(\mathrm{ia}(\mathrm{i}))+\mathrm{y}(\mathrm{ib}(\mathrm{i}))$, and automatically devise an optimized schedule of communication. CHAOS supports irregular assignment of data arrays to processors by using a globally accessible translation table to describe the location of elements of the array. The loop iterations are automatically partitioned (or repartitioned) and assigned to processors (based on trying to optimize the resulting load balance and communication volume). A preprocessing phase constructs the required communication schedules for the given distribution of workload and data.

DOLIB avoids the complexity of cache coherency by supporting a restricted virtual shared memory. Specifically, DOLIB assumes that any global array with caching enabled contains read-only data. If the array is updated, it is the programmer's responsibility to flush the cache to prevent erroneous results. In many important applications such as distributed finite element matrix assembly, parallel sparse matrix factorization and Lagrangian particle tracking, cache coherency is not an issue. For example, global data such as a flow field typically remains constant throughout a time-step for Lagrangian particle tracking. At the beginning of the next time step, the cache can be flushed to prepare for recomputing 
the flow field.

In section 4.1, we describe how caching is implemented in DOLIB, and discuss the DOLIB routines available to the user to control the cache.

\section{User Interface}

This section provides details on the syntax and behavior of each of the DOLIB primitives. They form the manual pages for DOLIB. 


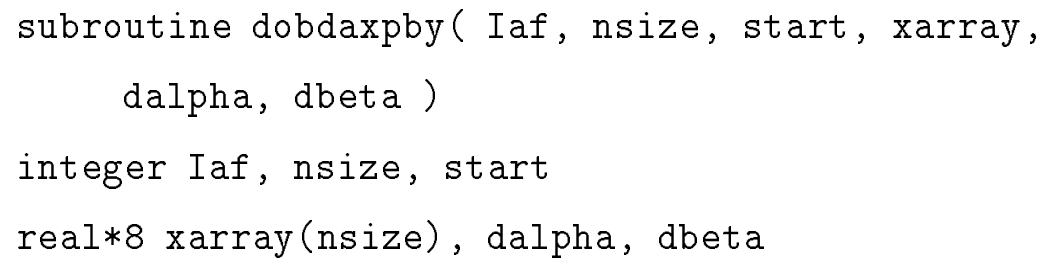

\section{Input parameters}

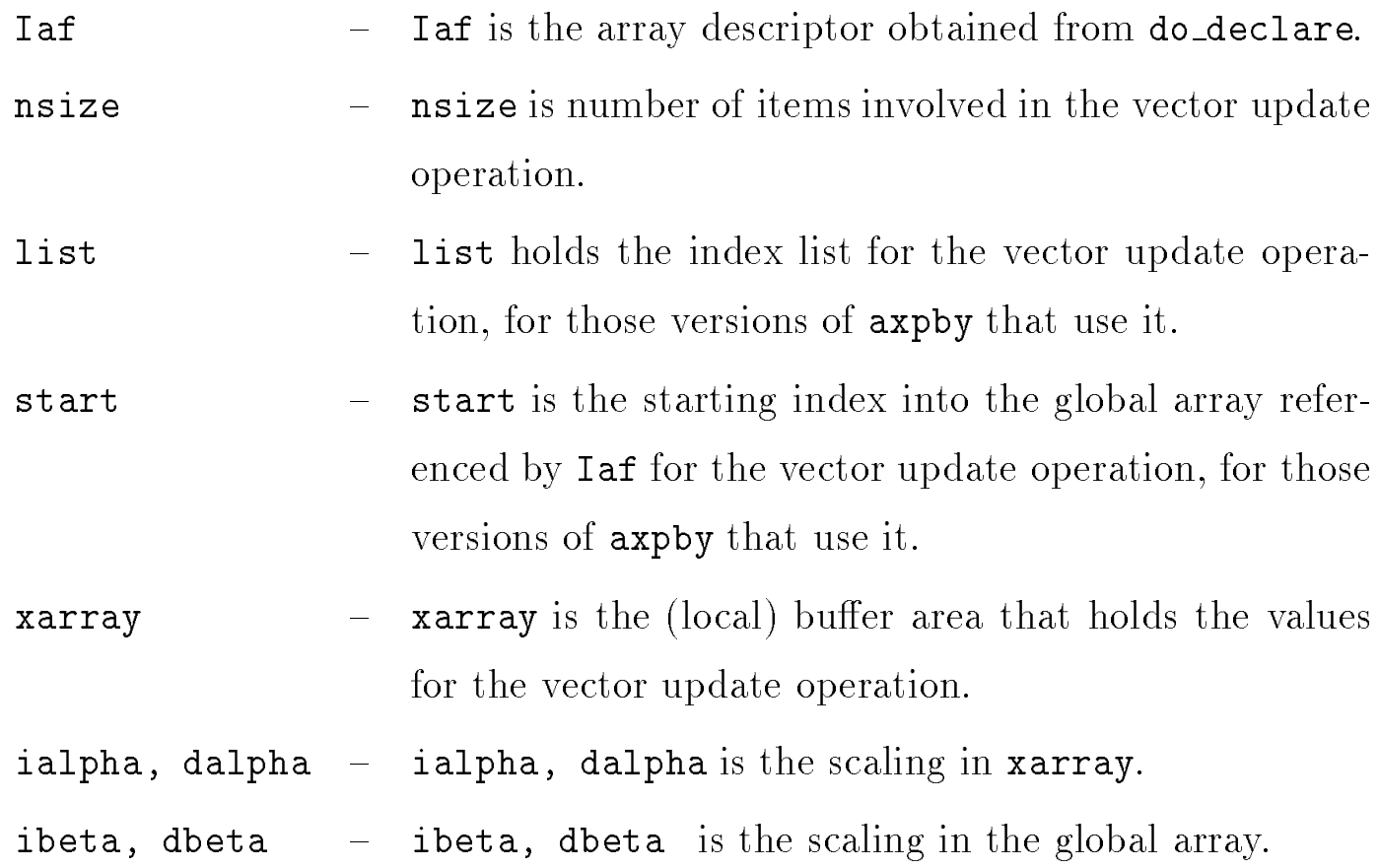

\section{Description}

do_axpby (and all of its variants) performs a distributed vector update operation into the global shared array out of the specified buffer. The index set is described in list (do_daxpby), or is derived as a contiguous block of nsize elements starting at index start (do_bdaxpby). It is an error to have nsize $\leq 0$.

The axpby operation can be used in assembly of finite element matrices into a global stiffness matrix. The accumulation of individual entries is performed as a critical section by the owner of the data page and hence eliminates the need for exclusive access or locking of the global array. 
Note that the scatter operation can be implemented as a special case of an axpby update with $\alpha=1, \beta=0$.

This is not a synchronous call. The calling process does not wait (block) until the axpby request is completed. Note that it is an error to access past the declared array size, gsize, set in do_declare. 


\section{do_declare}

do_declare allocates a distributed global shared array and returns a descriptor to the distributed object. An implicit global synchronization is performed.

\section{Synopsis}

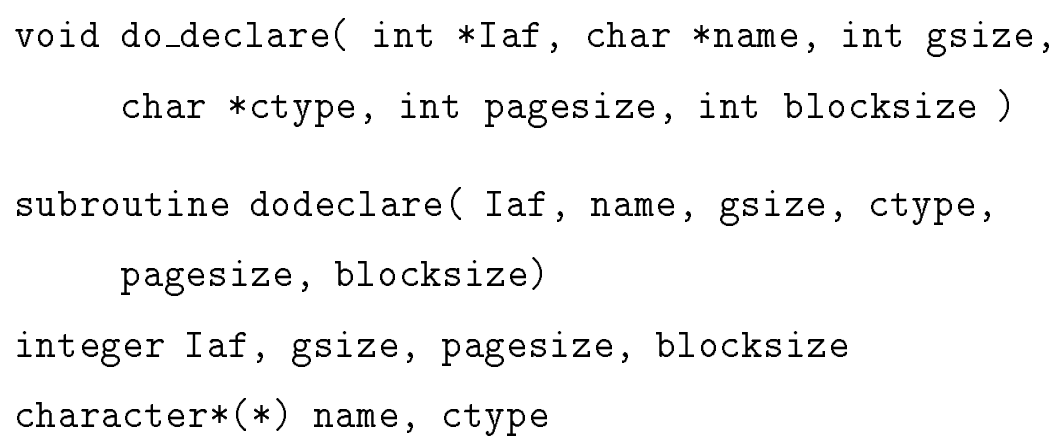

\section{Input parameters}

name $\quad-$ name is a null terminated character string that distinguishes the global array declared. It is used for displaying informative error messages. A null terminated string can be generated as name = "arrayname" // char $(0)$ in FORTRAN.

gsize - gsize is the number of items (not bytes) in the global array.

ctype - ctype is a null terminated character string that describes the type of the global array. Valid string values are "int" or "integer", "double", "real*8" or "double precision" and "char" or "character" (all either lower or upper case).

pagesize - pagesize is the number of items in a page. blocksize - blocksize is the number of pages in a block. Note there are blocksize*pagesize entries in a block. 


\section{Output parameter}

$$
\begin{aligned}
& \text { Iaf - Iaf is a descriptor to the global shared array. } \\
& \text { do_declare returns a positive value in Iaf on success. }
\end{aligned}
$$

\section{Discussion}

do_declare allocates a distributed global shared array and returns a descriptor (positive integer) to the distributed object. The array is composed of fixed-size pages grouped as blocks. If enabled, caching is performed on a page as a unit. The blocks are distributed among all processors in wrapmapped fashion. Actual storage is obtained by malloc() in the C language. Important note: indexing conforms to the conventions of the language being used. That is, a global array is indexed with 1:gsize in FORTRAN, $0:($ gsize-1) in $C$. The region of memory is not initialized in any way assume it is garbage.

All processors must participate in do_declare. An implicit global synchronization is performed. 


\section{do_destroy}

do_destroy deallocates global shared resources associated with the array descriptor. An implicit global synchronization is performed.

\section{Synopsis}

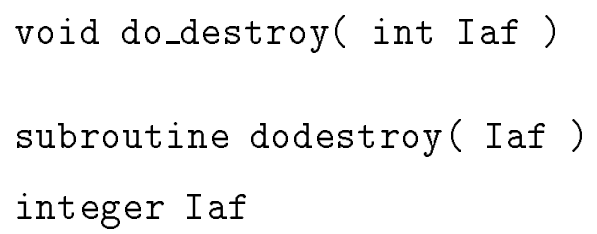

\section{Input parameters}

Iaf - Iaf is the array descriptor obtained from do_declare.

\section{Description}

do_destroy deallocates the global shared resources associated with the array descriptor Iaf. Memory is returned to the heap by free() in the $\mathrm{C}$ language.

All processors must participate in do_destroy. An implicit global synchronization is performed. 


\section{do_disable}

do_disable disables caching of data pages associated with the global array.

\section{Synopsis}

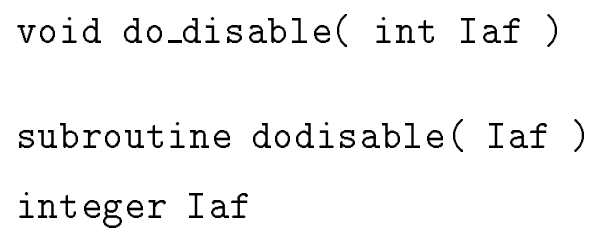

\section{Input parameters}

Iaf - Iaf is the array descriptor obtained from do_declare.

\section{Discussion}

DOLIB supports automatic caching of data pages to reduce communication and enhance performance. do_disable disables caching of data pages in the global array associated with descriptor Iaf. All data pages associated with global array descriptor Iaf in the cache are purged.

Important note: it is the programmer's responsibility to disable caching while the global array can be asynchronously updated. There is no implicit enforcement of cache coherency since only unmodified read-only pages should be cached.

Purging of data pages in the cache can be done by calling do_disableor by setting the cache size to be zero with do_setchsize. Caching can be turned on by calling do_enable. 


\section{do_wait}

do_wait blocks and waits till the asynchronous gather request is satisfied.

\section{Synopsis}

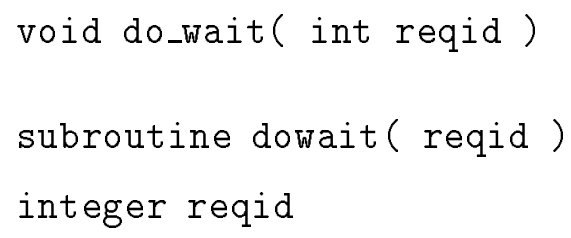

\section{Input parameters}

$$
\begin{aligned}
\text { reqid }- & \text { request descriptor returned from an asynchronous } \\
& \text { gather operation such as do_bgather or do_agather. }
\end{aligned}
$$

\section{Description}

do_wait blocks and waits until the data requested by an asynchronous gather has been copied into the user buffer. The request id reqid is released and invalidated after return from do_wait. Calling do_wait with an invalidated request id is an error. 


\section{do_enable}

do_enable enables caching of data pages associated with the specified global array.

\section{Synopsis}

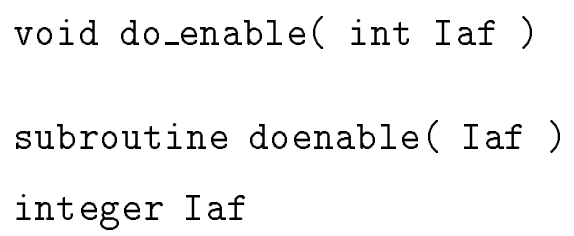

\section{Input parameters}

Iaf - Iaf is the array descriptor obtained from do_declare.

\section{Discussion}

DOLIB supports caching of commonly used data pages to reduce communication and enhance performance. do_enable enables caching of read-only data pages in the global array associated with descriptor Iaf. Caching is performed on a page (determined by pagesize in do_declare) as a unit. Important note: It is the programmer's responsibility to ensure only readonly unmodified data is cached. There is no implicit enforcement of cache coherency since only unmodified read-only pages should be cached. Although not required, a global synchronization (do_gsync) is strongly recommended before calling do_enable to ensure correctness.

Purging of data pages in the cache can be done by calling do_disableor by setting the cache size to be zero with do_setchsize. 


\section{do_gather}

do_gather performs a gather operation out of the global shared array into the specified buffer.

\section{Synopsis}

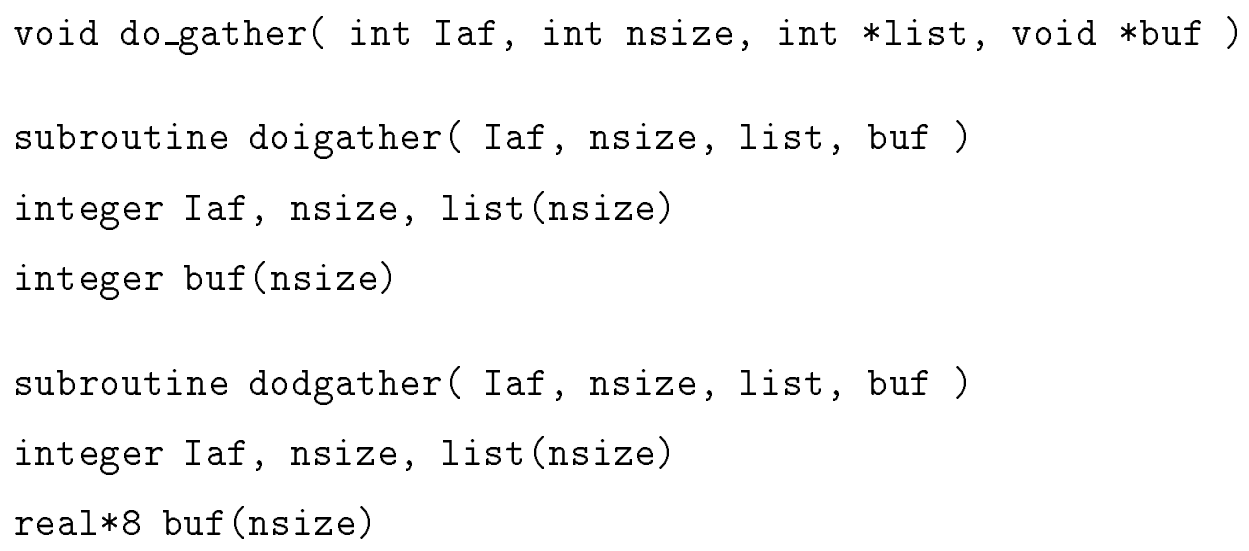

\section{Input parameters}

Iaf - the array descriptor obtained from do_declare.

nsize - number of items to be collected in the gather operation.

list - the index list for the gather operation.

buf - the buffer area to hold the result of the gather operation.

\section{Description}

do_gather performs a collect operation out of the global shared array into the specified buffer. The index set is described in list.

This is a synchronous call. The calling process waits (blocks) until the gather request is completed. Note that it is an error to attempt access beyond the declared array size, gsize, set in do_declare. 


\section{do_bgather}

do_bgather performs an asynchronous block gather operation out of the global shared array into the specified buffer.

\section{Synopsis}

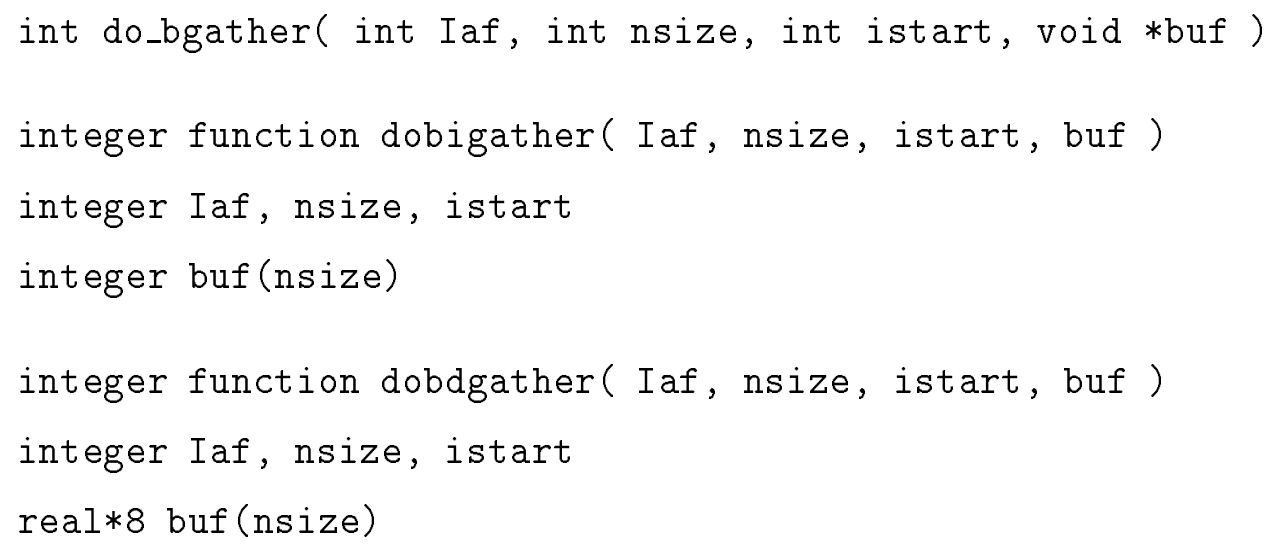

\section{Input parameters}

Iaf - the array descriptor obtained from do_declare. nsize - number of items to be collected in the gather operation. istart - the starting index for the gather operation. Indices range from istart to istart + nsize -1 are collected.

buf - the buffer area to hold the result of the gather operation.

\section{Description}

do_bgather performs an asynchronous block gather operation out of the global shared array into the specified buffer. The index set ranges from istart to istart + nsize -1 . This is an asynchronous call with the function returning an integer request descriptor to be used with do_is avail and do_wait. The buffer buf should be treated as invalid until either 
do_isavail returns .true. or 1 , or until do_wait is called. The calling process returns immediately without blocking. It is an error to attempt access beyond the declared array size, gsize, set in do_declare. 


\section{do_init}

do_init enables emulation of global shared memory. An implicit global synchronization is performed.

\section{Synopsis}



\section{Input parameters}



\section{Discussion}

do_init initializes the DOLIB library and enables the emulation of global shared memory. do_init must be called before any use of DOLIB routines. An implicit global synchronization is performed. 


\section{do_isavail}

do_isavail checks whether the data requested by an asynchronous gather are available.

\section{Synopsis}

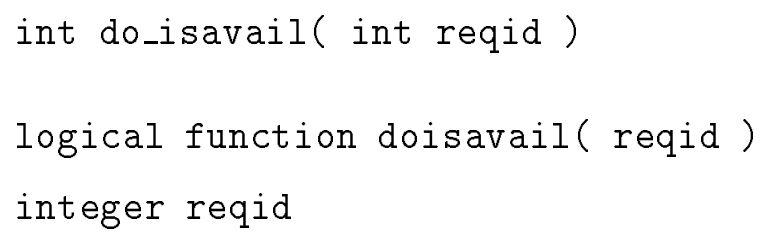

\section{Input parameters}

$$
\begin{aligned}
\text { reqid }- & \text { request descriptor returned from an asynchronous } \\
& \text { gather operation such as do_bgather. }
\end{aligned}
$$

\section{Description}

do_isavail returns .true. or 1 if the data requested by an asynchronous gather are available. The request descriptor reqid is released and is invalid as soon as do_isavail returns .true. or 1 . Calling do_isavail with an invalid request descriptor is an error. 


\section{do_scatter}

do_scatter performs a scatter (distributed copy) operation into the global shared array out of the specified buffer.

\section{Synopsis}

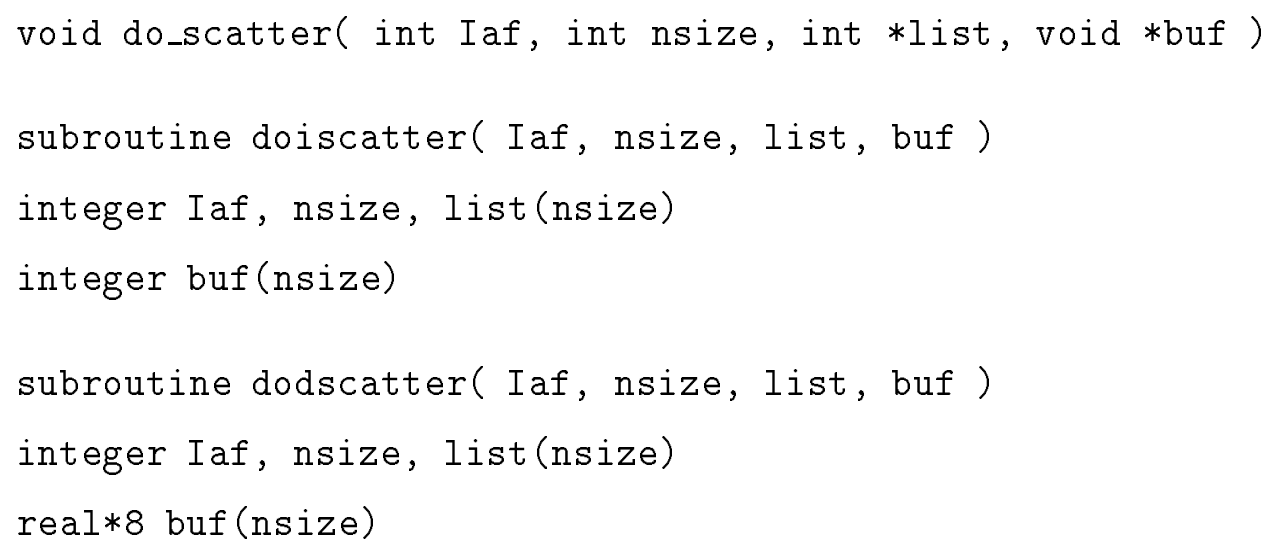

\section{Input parameters}

Iaf - the array descriptor obtained from do_declare.

nsize - number of items involved in the scatter operation.

list - the index list for the scatter operation.

buf - the buffer area that holds the values to be scattered.

\section{Description}

do_scatter performs a distributed copy operation into the global shared array out of the specified buffer. The index set is described in list. It is an error to have nsize $\leq 0$.

This is an asynchronous call. The calling process does not wait (block) until the scatter request is completed. Note that it is an error for do_scatter to attempt to access beyond the declared array size, gsize, set in do_declare. 


\section{do_gsync}

do_gsync performs an explicit global synchronization of all the processors. When the do_gsync operation is completed, all previously called DOLIB operations are guaranteed to have completed.

\section{Synopsis}

void do_gsync( )

subroutine dogsync

\section{Discussion}

The do_gsync routine synchronizes the processors and ensures that all active or pending DOLIB routines (including all do_gather, do_scatter, and do_axpby operations) have completed. do_gsync is useful for avoiding potential race conditions (such as when a do_scatter is immediately followed by a do_gather).

Application codes can freely mix DOLIB with other parallel programming primitives (such as PICL and PVM calls) when desired; however, before making calls to routines in these libraries, do_gsync should be called to purge processor buffers of any messages relating to DOLIB. 


\section{do_check}

do_check causes the calling processor to check its message queue for any pending DOLIB requests. If any are found, they are processed before do_check returns.

\section{Synopsis}

void do_check( )

subroutine docheck

\section{Discussion}

The do_check routine causes the calling processor to satisfy all pending DOLIB requests in its message queue. do_check is a no-op in the Intel multiprocessor version of DOLIB, since the interrupt handler causes all incoming requests to be processed. However, for the polling version of DOLIB (see section 6), do_check is provided so that the programmer can prevent a starvation condition or increase the frequency with which DOLIB requests are handled to enhance performance. 


\section{do_setchsize}

do_setchsize sets the size of the cache in terms of number of pages to be cached. do_setchsize returns the previous cache size.

\section{Synopsis}

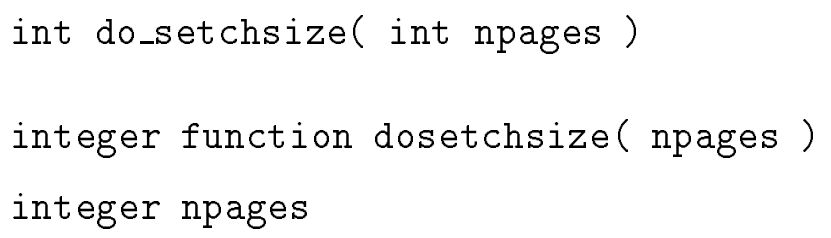

\section{Input parameters}

$$
\begin{aligned}
\text { npages } \quad \text { npages is the size of cache in terms of number of pages } \\
\text { to be cached. }
\end{aligned}
$$

\section{Discussion}

DOLIB supports automatic caching of commonly used data pages to reduce communication message traffic and enhance performance. A common shared cache pool is used even though page size for each global array may be different. A larger cache would yield a better "hit ratio" but require more memory and entail a higher overhead for associative searching. The programmer can set the cache size appropriate for a specific application for optimal performance.

Note setting the cache size to be zero is a fast way of purging the entire cache. 


\section{Implementation Details}

DOLIB is designed to provide support for globally shared arrays in a distributedmemory environment. In this section, we describe some of the design decisions made that may affect the performance of application codes that use DOLIB.

DOLIB views a large global array as composed of fixed size pages stored in a block wrapped fashion across all processors. This page structure simplifies caching, which is vital for good performance. The restriction to a block wrapped mapping allows DOLIB to easily find the location (processor number and machine address) of any array element. Pages can be easily malloc'ed or free'ed.

DOLIB for the Intel iPSC/860 and Paragon machines is implemented using the IPX (Inter Process eXecution) [5] system developed at Brookhaven National Laboratory. ${ }^{1}$ This version of DOLIB (and IPX) relies heavily on a reliable interrupt mechanism provided by hrecv on Intel multiprocessors. If a processor makes a call to do_gather, DOLIB first determines where (on which other processors) the requested data reside. For example, suppose that processor A requires data residing on processors $\mathrm{B}$ and $\mathrm{C}$. do_gather causes processor A to send message requests that interrupt processors $\mathrm{B}$ and $\mathrm{C}$ from regular computation. These processors package the requested data and send reply messages back to Processor A. They then exit this "interrupt" mode and resume regular computation. At no time is the thread of regular computation "aware" of the interruption. However, when the interrupt mode is entered and assumes control of the processor, there may be two detrimental effects on the regular computation. First, data that reside in the hardware cache may be discarded during interrupt processing, thus increasing memory access time when regular computation is resumed. Second, if the regular computation involves pipelined floating point operations, the pipeline will be interrupted, thus increasing the overhead in reloading the pipe when regular computation is resumed.

The do_scatter operation involves a similar sequence of messages as the

\footnotetext{
${ }^{1}$ IPX is available by anonymous FTP from the site msg.das.bnl.gov under the directory /pub/ipx.
} 
do_gather.

The important facility provided by Intel's hrecv primitive is that all such signals are caught. For example, if processor $\mathrm{B}$ in the example above receives another interrupt while processing the one from processor $A$, the new interrupt is queued and then processed before processor B returns to normal execution mode. We will discuss these operations in more detail later in this section.

We have also developed a version of DOLIB (based on a polling version of IPX) that does not require a preemptive interrupt mechanism. In section 6 , we discuss the implementation of DOLIB in the absence of such reliable signal handling.

DOLIB must be initialized with a call to do_init before any other DOLIB calls can be made. The DOLIB routine do_declare defines a new global shared array. The user provides: the global array size (total number of elements); the data type (double or real*8, int or integer, char or character); the number of data items per page (page size) and the number of pages per block (block size); and a name for the array (to allow for useful error messages), though accessing the array is always done through the array descriptor that is returned from do_declare. Since memory for the new global array is immediately allocated and the values of the resulting local data addresses are shared among the processors, do_declare implicitly synchronizes the processors. Thus, all processors must participate in the do_declare operation.

\subsection{Caching}

Caching is disabled by default when an array is declared. The DOLIB routine do_enable enables caching of the indicated array. There is a single cache for all the global arrays that have had caching enabled. The DOLIB routine do_setchsize can be used to specify the maximum size (number of cache pages) in the cache, which currently defaults to 128 . The do_setchsize function returns the previous cache size. Note that for a given amount of memory there is a tradeoff between the number of cache pages allowed and the size of each page in the array. Both are chosen at run time, allowing the user to optimize the use of the cache for the 
given application. Moreover, since the pagesize of a global array is set when it is declared, the pages in the cache may be of different sizes. For the Intel iPSC/860 and Paragon machines, a page size of 8 Kbytes is reasonable.

Important note: Some patterns of accessing a global shared array may be unexpectedly memory-intensive. For example, consider a do_gather operation in which a single element of every page in the array is requested. The page containing each element is returned to the requesting processor, causing a copy of the entire global array to be temporarily created. For large arrays, such an operation may overflow the available memory, causing an error.

DOLIB makes no attempt to maintain cache coherency across processors. It is the user's responsibility to ensure that any information that is cached is read-only. The user may flush the cache pages associated with any given array by calling do_disable. No implicit synchronization is done in do_disable, though the utility of selectively disabling caching of an array on some processors is unclear.

To illustrate the utility of do_enable / do_disable, consider the problem of tracking particles along characteristics of a changing flow field, where the flow field is stored in a global shared array. Throughout a given time step, the flow field is assumed constant (and hence, read-only data). After all particles have been tracked in the given time step, the flow field must be updated for the next time step. Since the flow field is no longer read-only data, the cache should be purged of the flow field by calling do_disable. After the new flow field has been computed and stored in the global array, caching can be re-enabled for the flow field with do_enable.

To purge the entire cache without the necessity of disabling caching on each global array individually, the user can simply set the cache size to 0 using do_setchsize (the previous value is returned by the function), and then reset it to its previous value. 


\subsection{Accessing a Global Shared Array}

The main DOLIB routines that access the globally shared arrays are do_gather, do_scatter and do_axpby. The do_axpby routine implements the operation $y \leftarrow$ $\alpha x+\beta y$, where $\alpha$ and $\beta$ are constants, $y$ is a globally shared array in DOLIB, and $x$ is a local vector. do_axpby is a powerful and flexible primitive, and is commonly used in such contexts as finite element matrix assembly.

When a processor (say, processor A) calls the do_gather routine requesting a list of elements from a global shared array, the index list is processed to determine where the requested data reside. (If one of the contiguous block versions is called, the starting index and number of items is treated similarly). Items that are not local to processor A are obtained from other processors using the IPX get_array call. The information exchanged among the processors during the do_declare allows processor $\mathrm{A}$ to compute the machine address on the remote processors where the data reside. Using this address, the get_array call interrupts the remote processor, forcing it to return a message containing a copy of that data.

DOLIB provides both synchronous and asynchronous versions of the gather operation. The synchronous version causes the calling processor to block until the gather has been completed. The asynchronous version returns control immediately to the calling processor, providing a request descriptor as return value. The calling processor can then query the status of the asynchronous gather using do_isavail (with the request descriptor) to determine whether the gather has been completed. Calling do_wait will force the processor to block until the specified gather has been completed. The asynchronous call is provided to allow

for overlapping of communication with computation; however, the user should be aware that there is some overhead associated with creating a request descriptor and allocating memory to contain the returned pages. The asynchronous version may be more expensive overall in cases where there is little chance of overlapping communication with computation.

Given a list of values for the local array $x$ and a corresponding list of array indices for the global array $y$, do_axpby accumulates the updated values to the 
appropriate location in the global array. Global array references that reside on the calling processor (say, processor A) are done directly by assignment to memory; the others require message passing, as follows: Processor A determines the owner processor id, block number, page number and page offset for each array reference, grouping those belonging to the same processor for efficiency. Then $\mathrm{A}$ sends an interrupt message to each such processor (say, B and C), followed by a message containing the necessary values and indices. The interrupt message induces processors $\mathrm{B}$ and $\mathrm{C}$ to complete the do_axpby operation with the values contained in the second message.

The procedure for implementing do_scatter is identical to that of the do_axpby routine. Indeed, the DOLIB do_scatter routine is simply a do_axpby call with $\alpha=1$ and $\beta=0$.

Warning: If a do_scatter operation is immediately followed by a do_gather operation, a race condition may ensue, and old values (before the do_scatter has completed) may be returned to the do_gather call. A do_gsync should be called in between the do_scatter and do_gather to prevent this type of error.

\subsection{Reclaiming Memory}

One major advantage of DOLIB is the ability to dynamically create and destroy global shared arrays. When an array is no longer needed, do_destroy will free all memory allocated to the array, including cache pages associated with the given array. To avoid inconsistent views of the array, the processors are first synchronized (to ensure that any outstanding gather requests are satisfied), and then the data structures for the array are destroyed. This implies that all processors must participate in the do_destroy operation.

\section{Performance of DOLIB on Intel iPSC/860}

DOLIB is currently implemented using the IPX message system developed at Brookhaven National Laboratory. IPX relies heavily on the hrecv() preemptive 
interrupt capability on Intel multiprocessors. Access cost to emulated shared memory is due in part to the overhead in DOLIB, IPX and the underlying NX message delay. In this section, we illustrate the performance and use of DOLIB in the context of a parallel matrix-matrix multiply algorithm. The RATFOR source is included in the Appendix.

The algorithm computes the matrix product $C=A * B$ where matrix $A$ is $N \times M$ and $B$ is $M \times M$ on $P$ processors. The computation proceeds by block row partition of $\mathrm{A}$ among processors. Block columns in matrix $B$ are gathered to generate parts of matrix $C$ by calling the BLAS routine dgemm. The volume of communication for each processor is $O(N M / P)$ for gathering matrix $A, O\left(M^{2}\right)$ for gathering $B$ and $O(N M / P)$ for scattering results back to matrix $C$.

Tables 5.1 and 5.2 display the run time (in seconds) on an Intel iPSC/860 with 8 Mbytes of memory on each node. The run times are measured by calling the NX dclock() routine. The items gathA, gathB, scattC, dgemm denote the maximum communication time among all processor to gather $A$, to gather $B$ to scatter $C$ and the computation time in the level 3 BLAS dgemm. The total time is the elapsed time from a gsync() at the start of the algorithm to another gsync() at the end.

The matrix dimension $N$ affects the data distribution of the global matrices $A$ and $C$ since data pages are assigned to processors in a wrapped fashion. If $N$ is exactly divisible by $P(\bmod (N, P)=0)$, then all references to matrices $A$ and $C$ are satisfied locally on each processor without external communication. This is reflected in the faster times for the gather of $A$ and scatter of $C$ for $N=1440,19200$ in Table 5.1 and $N=1920,37440$ in Table 5.2.

\section{Implementation of DOLIB using Polling}

The version of DOLIB for the Intel iPSC/860 and Paragon machines relies heavily on the reliable interrupt handling capabilities of Intel's multiprocessors. We have implemented a version of DOLIB that relies on explicit polling of the message queue to service DOLIB requests. However, there are several requirements imposed on 
Table 5.1: 16 nodes on ipsc/860

\begin{tabular}{|c|c|c|c|c|c|}
\hline \hline$(\mathrm{N}, \mathrm{M})$ & gathA & gathB & scattC & dgemm & total time \\
\hline \hline$(1440,1440)$ & 1.1 & 15 & 2.1 & 12 & 36 \\
$(1441,1441)$ & 4.2 & 16 & 4.2 & 12 & 42 \\
$(19200,160)$ & 0.13 & 1.0 & 1.2 & 1.9 & 4.2 \\
$(19201,160)$ & 2.7 & 1.4 & 2.0 & 2.0 & 7.9 \\
\hline
\end{tabular}

Table 5.2: 32 nodes on ipsc/860

\begin{tabular}{|c|c|c|c|c|c|}
\hline \hline$(\mathrm{N}, \mathrm{M})$ & gathA & gathB & scattC & dgemm & total time \\
\hline \hline$(1920,1920)$ & 1.5 & 29 & 2.9 & 15 & 61 \\
$(1921,1921)$ & 6.2 & 30 & 12 & 15 & 75 \\
$(37440,160)$ & 0.14 & 1.2 & 1.2 & 2.0 & 4.4 \\
$(37441,161)$ & 3.6 & 2.3 & 2.1 & 1.9 & 9.2 \\
\hline
\end{tabular}

the user of this version of DOLIB.

First, every DOLIB routine starts by checking its receive queue for DOLIB requests that must be satisfied. Hence, as long as all processors are executing DOLIB routines on a regular basis, performance should be largely unaffected. However, if any of the processors fails to call DOLIB routines, it will not check its receive queue for DOLIB requests, thus starving any processors attempting to access its array elements. Note that this is not a problem under the Intel version of DOLIB, since the interrupt handler forces the processor to handle incoming requests. The do_check routine is provided to allow the programmer to force a processor to check its input queue for DOLIB requests. The do_check routine is a no-op under the Intel version of DOLIB.

Our initial port of DOLIB to PVM (using a translation of IPX) is complete; however, because the IPX get_array routine is unaware of data types and treats all arrays as a sequence of bytes, DOLIB assumes a consistent integer and double precision format. Hence, currently only heterogeneous networks containing machines that agree on these formats (such as Sun Sparcstations and IBM RS/6000's) are 
supported. We are currently rewriting DOLIB to use the PVM3.3 message passing library directly. When this is completed, DOLIB will provide a shared-memory programming paradigm for a wide variety of platforms, including fully heterogeneous clusters of workstations.

\section{Summary}

We have described the design and implementation of DOLIB, a library of routines that support virtual shared memory on the Intel family of distributed memory machines. DOLIB provides access to globally shared arrays through the explicit use of gather and scatter primitives. Globally shared arrays are dynamically created and destroyed by the user application, thus allowing efficient use of the available aggregate memory on the multiprocessor. DOLIB also supports automatic caching of read-only memory, to increase the efficiency of global shared memory by reducing the amount of message passing required.

We provided two illustrations of the use of global shared memory: a matrixmatrix multiplication routine, and faster concurrent I/O using a library called DONIO. These examples show that global shared memory can be an effective means of providing the shared-memory programming paradigm on distributed memory machines.

\section{Obtaining the Software}

To obtain the source code for DOLIB, the reader should send email to the authors: efdazedo@msr.epm.ornl.gov or rominech@ornl.gov.

\section{Acknowledgements}

The authors would like to express appreciation to Bob Marr, Ron Peierls and Joe Pasciak for the IPX package, which simplified the development of DOLIB. 


\section{Appendix}

In this appendix, we list FORTRAN source code to illustrate the use of the DOLIB primitives. The source code given here is for the matrix-matrix multiplication example discussed in section 5 .

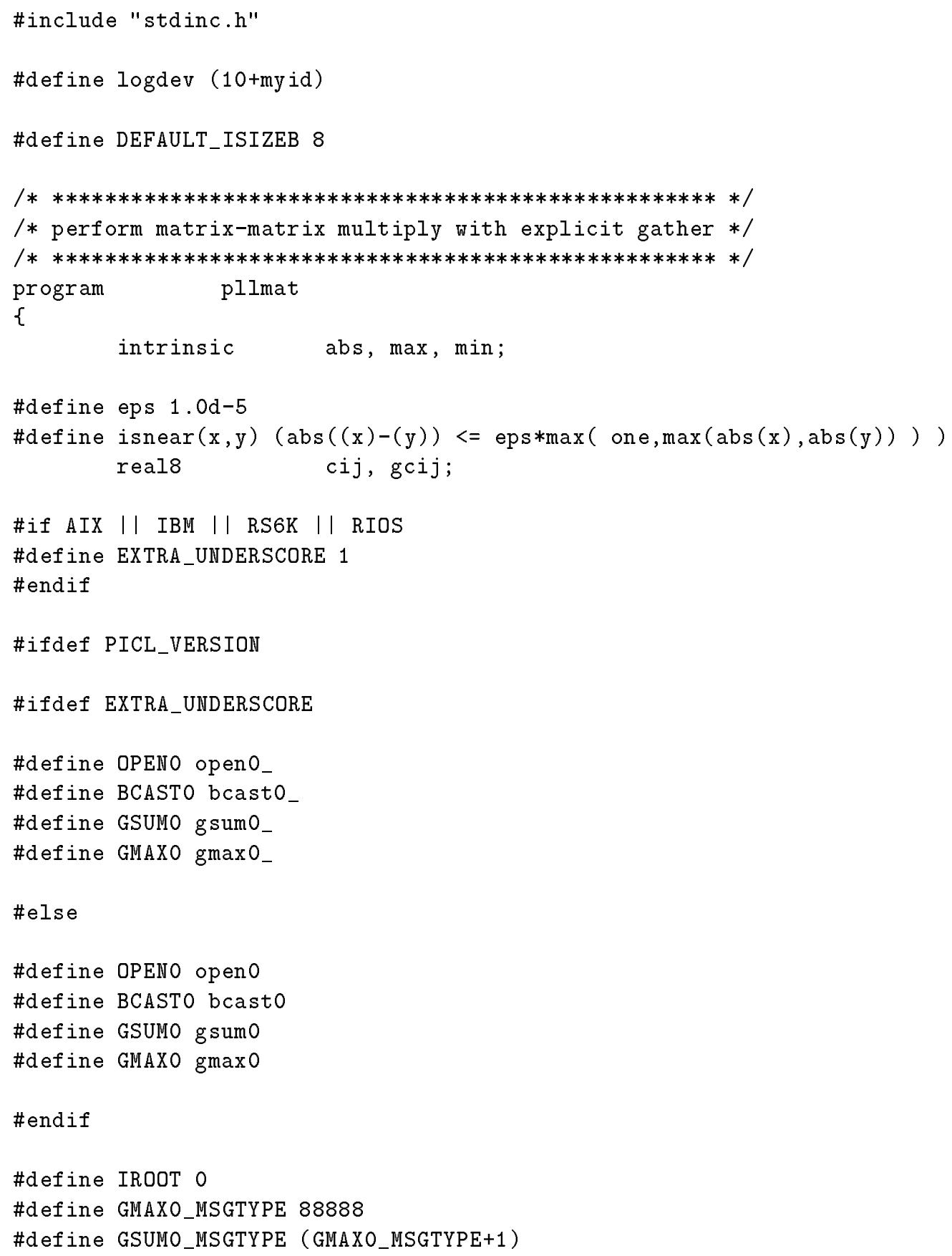









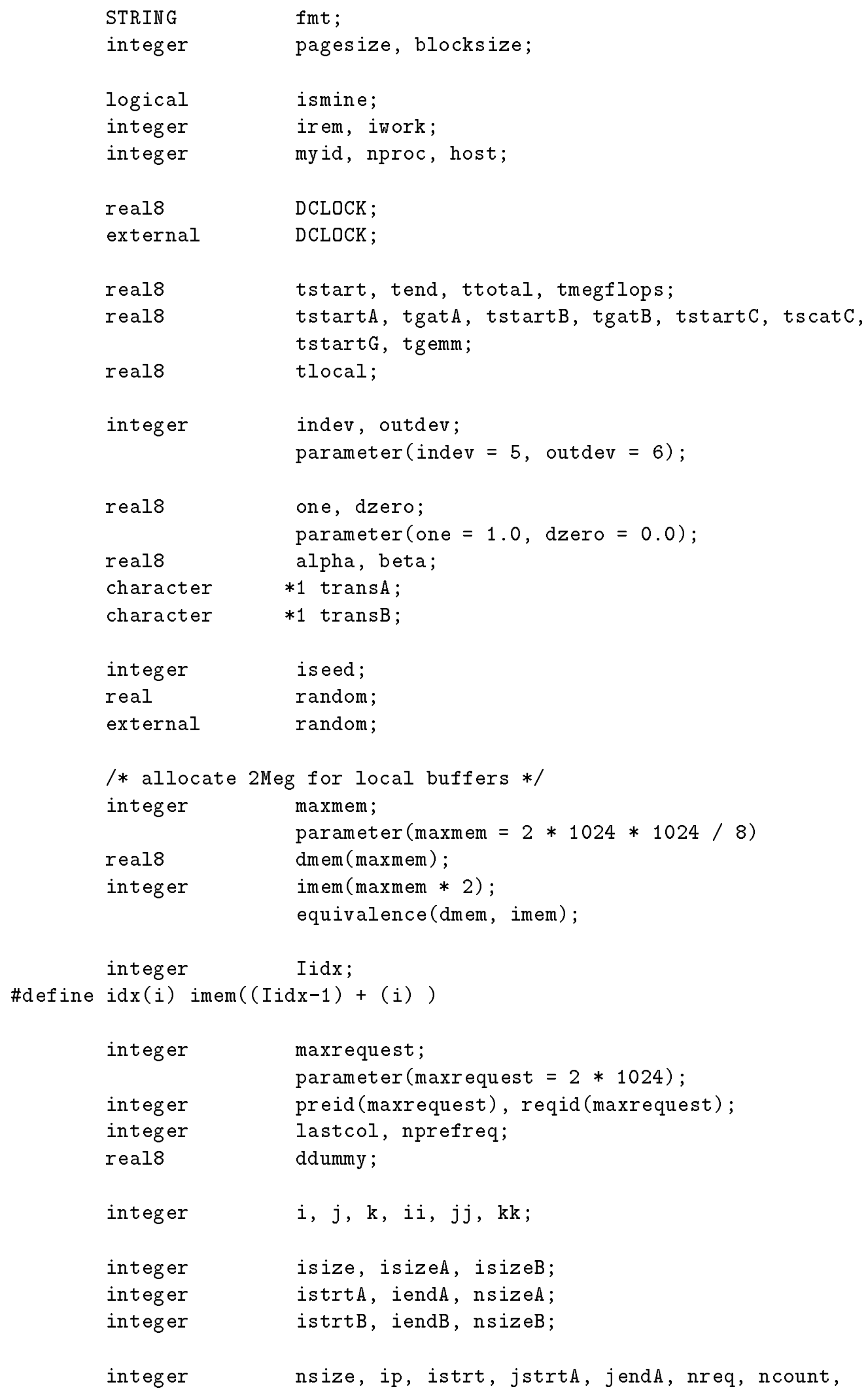




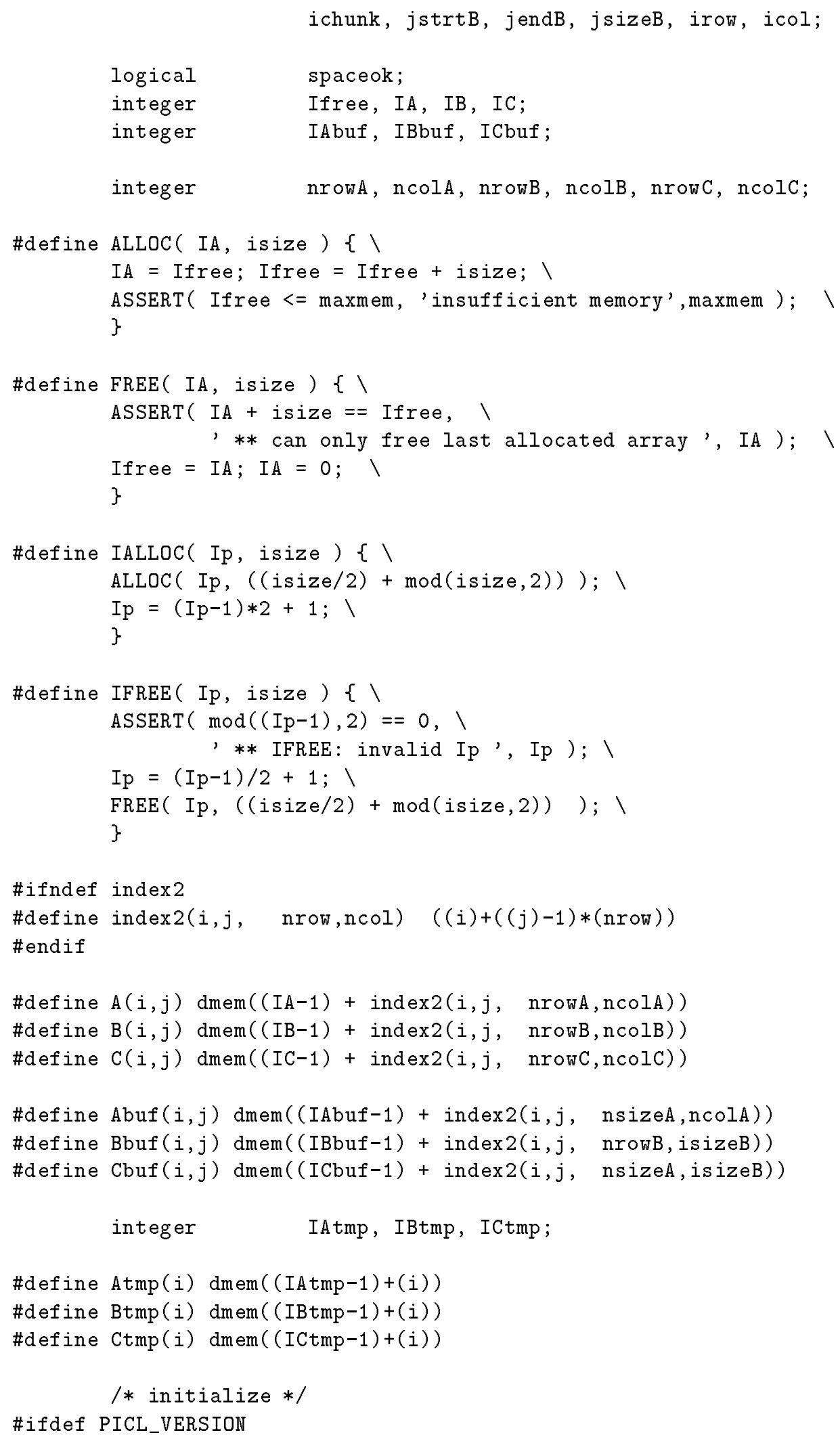




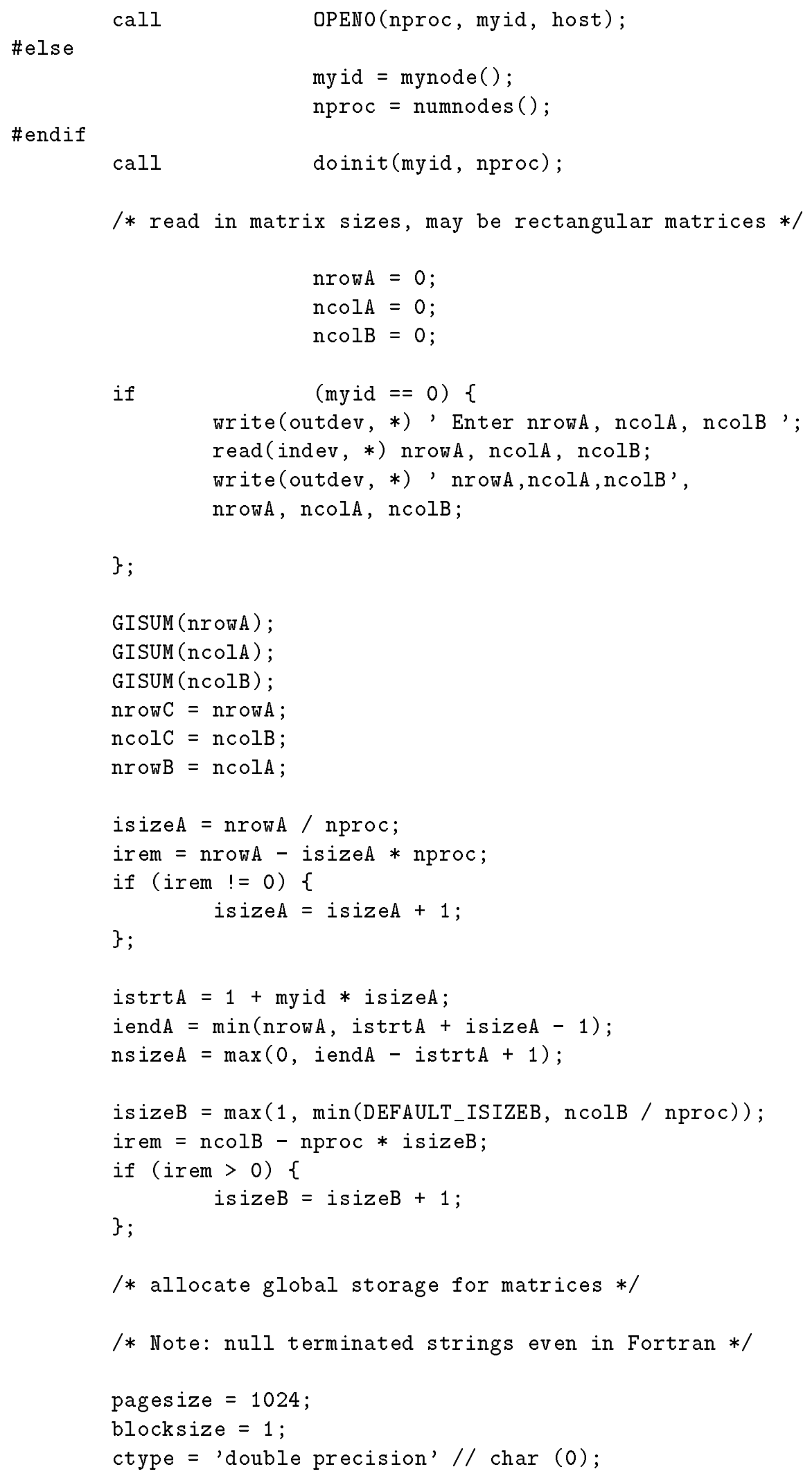




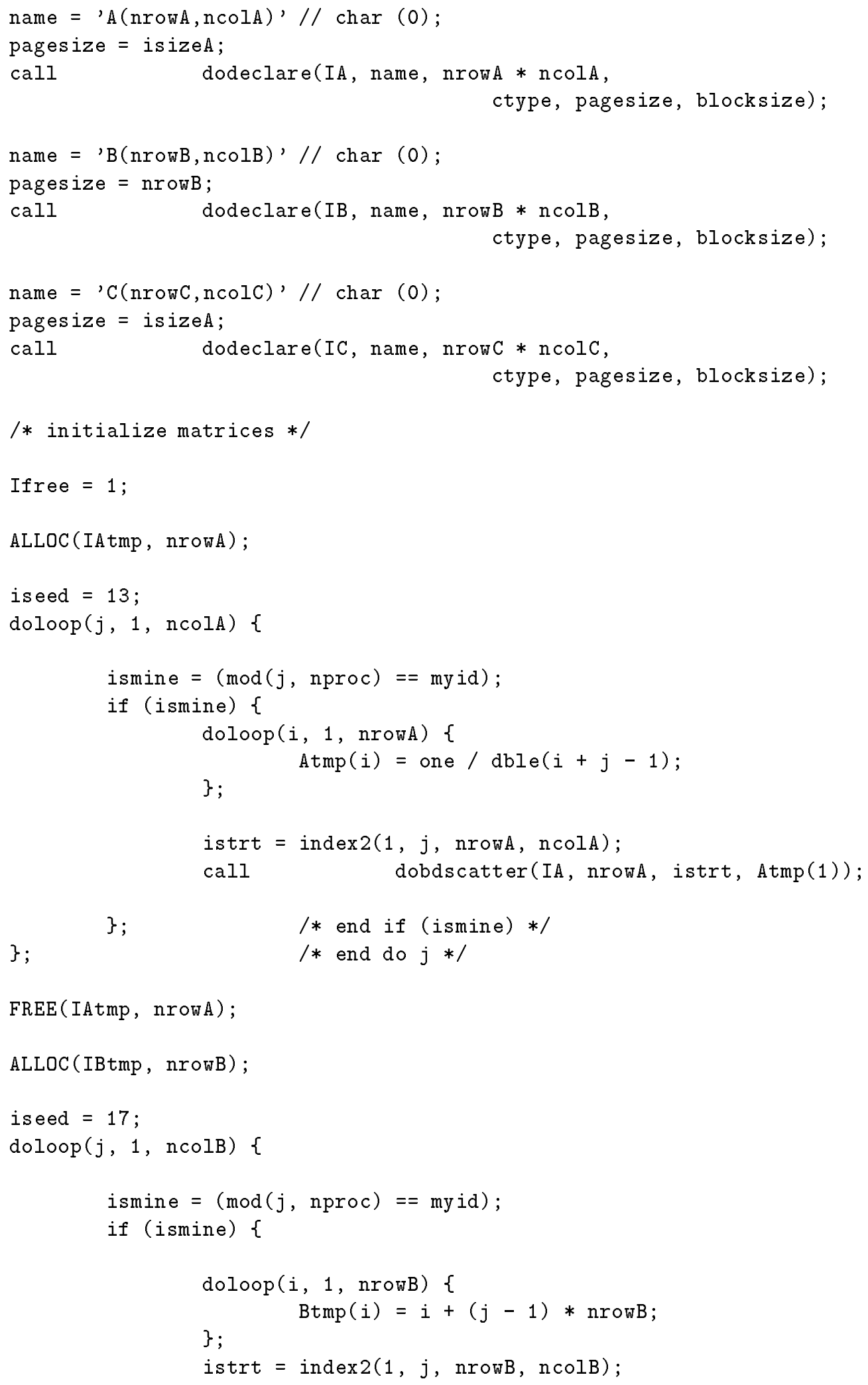


call dobdscatter(IB, nrowB, istrt, Btmp(1));

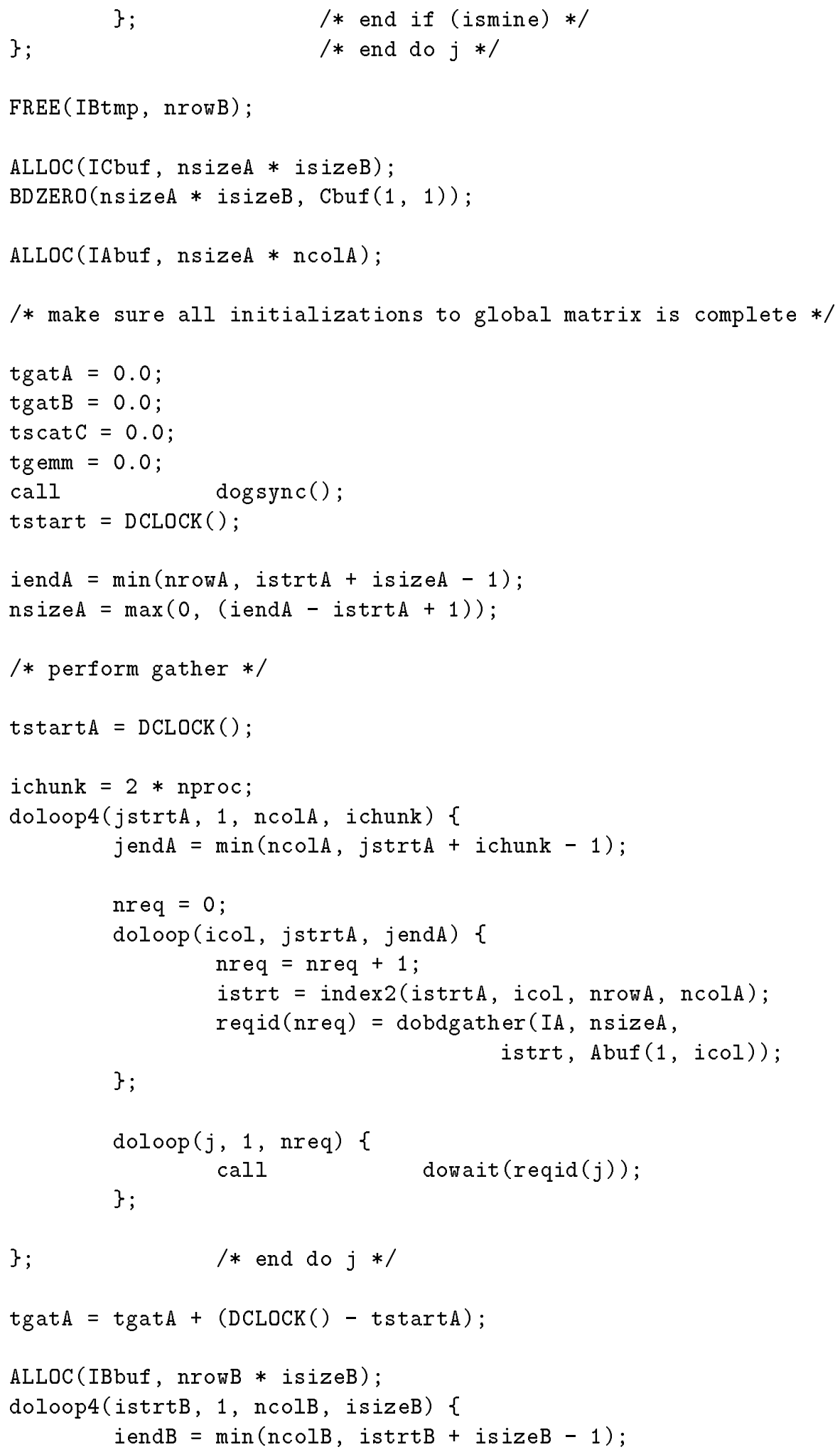




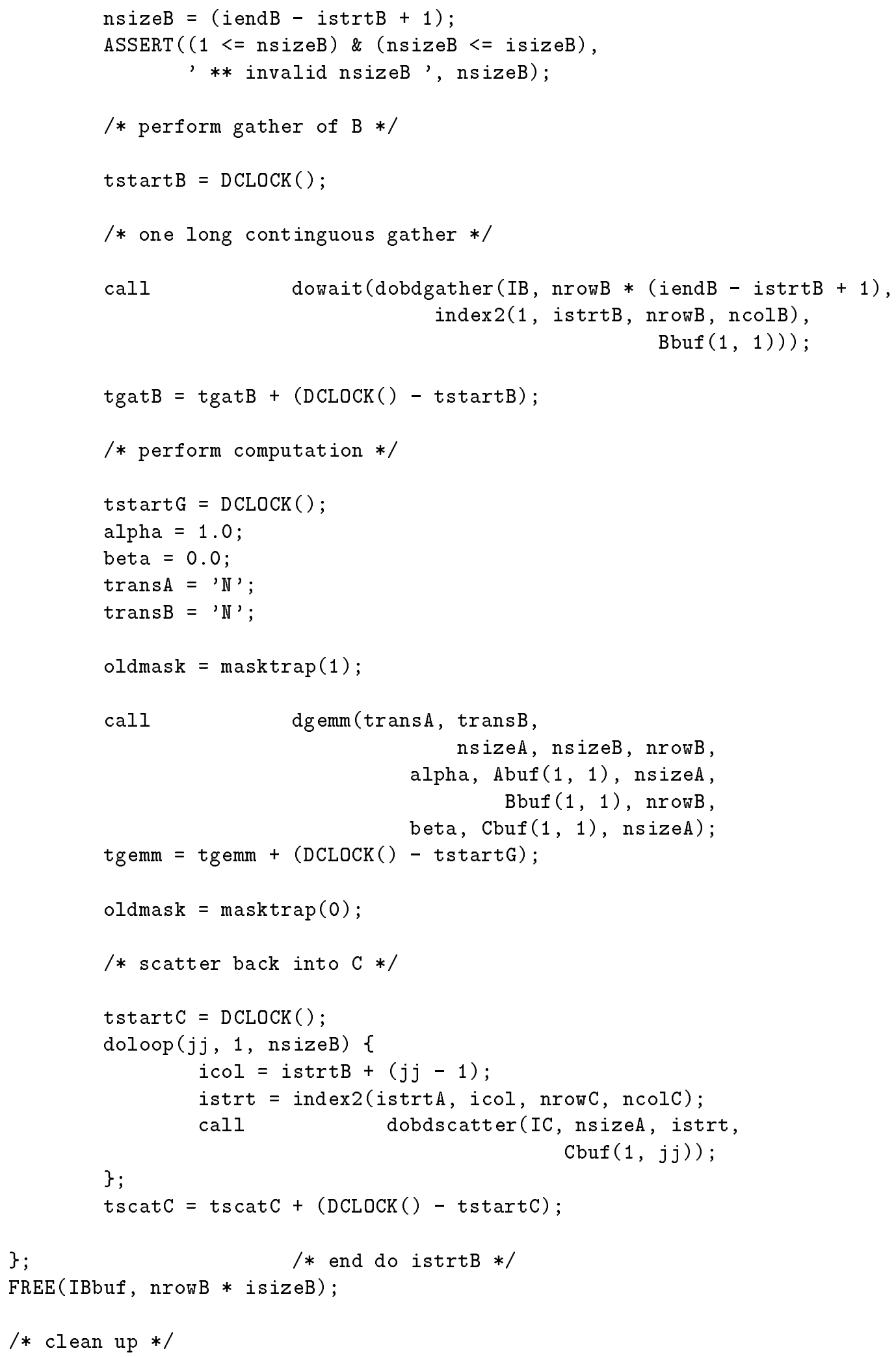




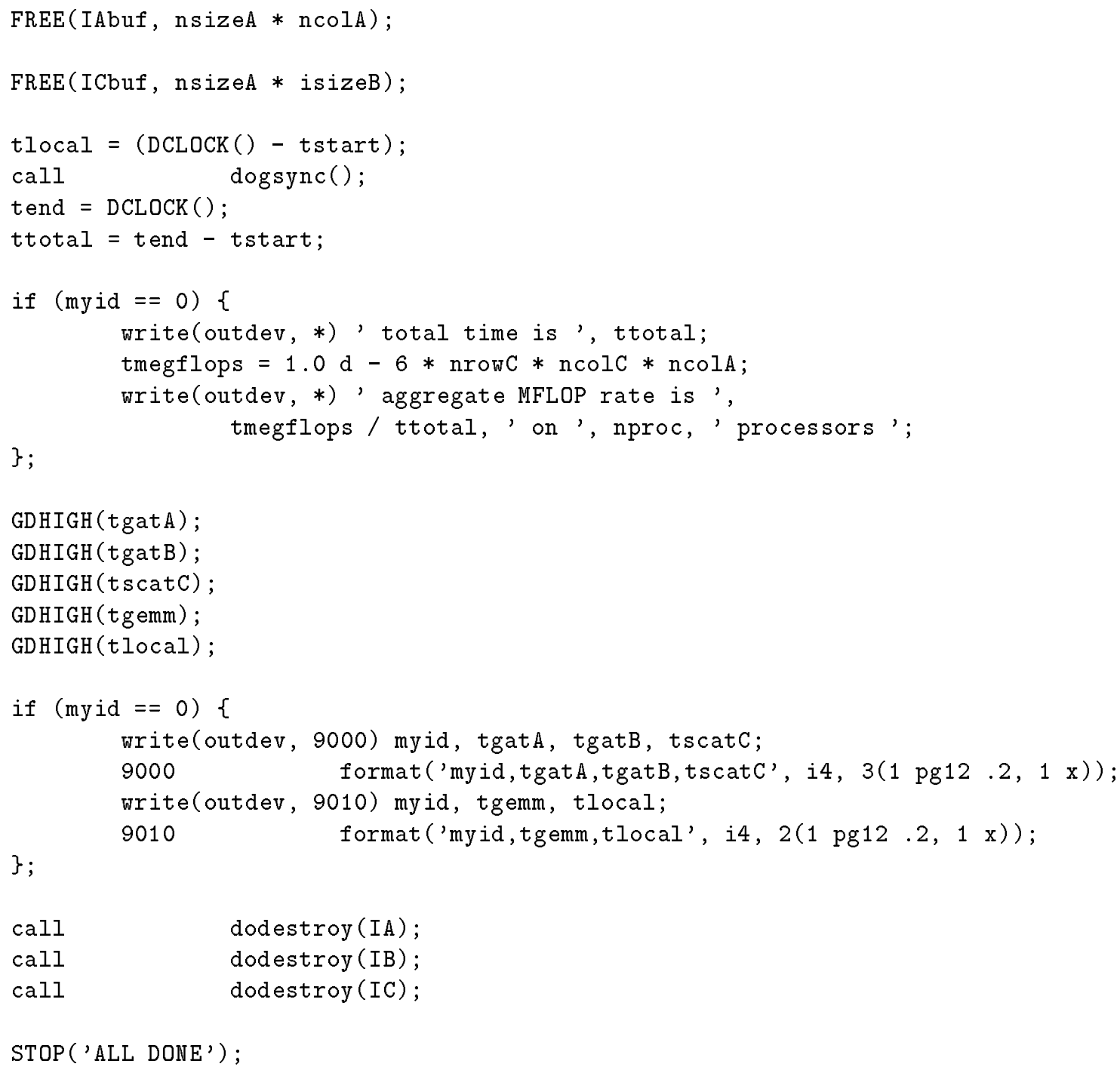




\section{References}

[1] E. F. D'Azevedo And C. H. Romine, DONIO: Distributed object network I/O library, Tech. Report ORNL/TM-12743, Oak Ridge National Laboratory, 1994.

[2] K. LI, Shared Virtual Memory on Loosely Coupled Multiprocessor, PhD thesis, Yale University, 1986.

[3] K. LI AND P. HUDAK, Memory coherence in shared virtual memory systems, ACM Transactions on Computer Systems, 7 (1989), pp. 321-359.

[4] K. LI AND R. SCHAEFER, Shared virtual memory for a hypercube multiprocessor, in The Proceedings of the Fourth Conference on Hypercubes, Concurrent Computers and Applications, March 1989, Monterey California, Golden Gate Enterprises, Los Altos, California, 1989, pp. 371-378.

[5] B. Marr, R. Peierls, and J. Pasciak, IPX - Preemptive remote procedure execution for concurrent applications, tech. report, Brookhaven National Laboratory, 1994.

[6] S. Sharma, R. Ponnusamy, B. Moon, Y.-S. Hwang, R. Das, and J. SALTZ, Run-time and compile-time support for adaptive irregular problems. Submitted for Publication.

[7] M. Stumm And S. Zhou, Algorithms implementing distributed shared memory, IEEE Computer, (May 1990), pp. 54-64. 